\title{
Embedded Control System for a Powered Leg Exoskeleton
}

\author{
Christian Fleischer and Günter Hommel
}

Institute for Computer Engineering and Microelectronics

Berlin University of Technology, Germany

fleischer@cs.tu-berlin.de

hommel@cs.tu-berlin.de

In this paper an embedded control system for a powered orthosis is presented. The orthosis (as shown in Fig. 1) is used to support the thigh muscles during flexion and extension of the knee while performing common motions like getting up from a chair, walking, or climbing stairs. The user interface of the control system is implemented by evaluating EMG signals from significant thigh muscles to find out the intended motion of the subject. The intended motion is executed with a linear actuator to support the subject's own muscle force.

Results from two experiments are presented. During the trials the torque support from the actuator illustrates the performance of the system.

\section{Introduction}

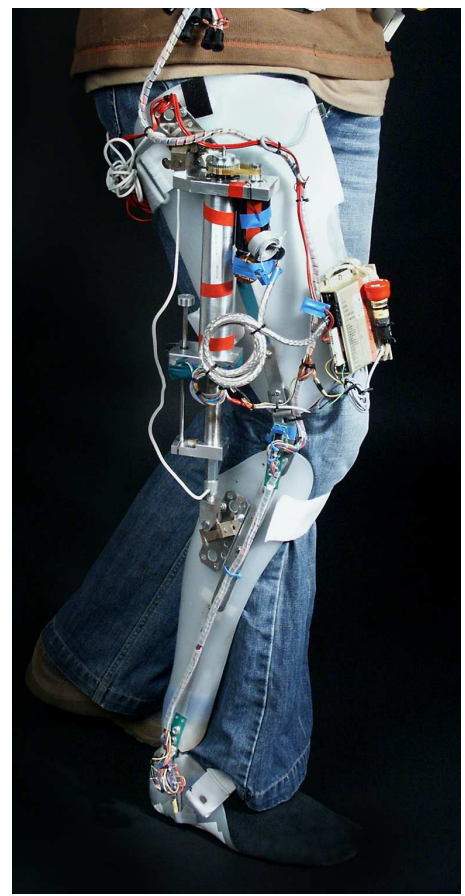

Fig. 1. The exoskeleton for knee support.

Exoskeleton systems for human operators offer a wide range of possible applications: For patients they can offer assistance during their rehabilitation process by guiding motions on correct trajectories to help re-learning motion patterns, or give force support to be able to perform certain motions. In factory environments they could remove load from workers to avoid wearing 
down their bodies through strenuous physical work. Depending on the size, weight, and handling of the devices, they could even be beneficial in everyday life at home, especially for elderly people.

Exoskeletons also offer a unique way of giving force feedback to the human body. They can act as haptic interfaces for telemanipulation, games and entertainment, or muscle and motion training devices for athletes.

Since numerous applications for exoskeletons can be imagined, many groups have shown interest in this topic. Especially in recent years several projects have emerged: The Berkley Lower Extremity Exoskeleton (BLEEX) for example is a military exoskeleton to aid soldiers carrying heavy loads [1]. The Hybrid Assistive Leg (HAL) is an actuated body suit for both legs [2], in the latest version (HAL-5) extended for both arms. It is designed for multiple purposes, such as supporting elderly people or as rehabilitation device. The powered lower limb orthosis described in [3] is developed to assist during motor rehabilitation after neurological injuries by re-learning gait patterns.

Whatever the use of such an exoskeleton is, there is need of an interface between the device and the person as soon as interaction is desired (no mere repetition of pre-defined trajectories).

Since standing, walking, and climbing stairs while keeping a stable pose are complex and very dynamic tasks, activating pre-defined motion patterns is not possible since perturbations from outside (contact forces) or the operator himself (swinging with the arms, leaning forward etc.) will interfere with the stability of the pre-calculated motions. So it is necessary to develop an intuitive interface that allows continuous control of the exoskeleton. In most of the developments one out of two alternatives is chosen: Either the desired motion is recognized by force sensors between the human and the mechanical construction or bio-signals are taken directly from the subject. The differences between the approaches are found in the underlying interpretation of the signals and the control algorithms.

Beside the standard application of EMG signals to analyze muscle activation and functionality during a rehabilitation process or analysis of diseases, more focus has recently been put on controlling robot arms and exoskeletons with those signals $[4,5,6]$. In Lloyd [7] a promising but very complex musculoskeletal model is presented that takes into account 13 muscles crossing the knee to estimate the resulting knee torque that could be used to control an exoskeleton, although the analysis presented there was not performed in real-time.

In [8] EMG signals of the Biceps Brachii and Triceps Brachii where used to estimate the elbow joint moment. With this estimation a moment controller was fed to allow control of a two-link exoskeletal arm to lift an external load with the hand. This approach is very similar to the one described here, but applied to a different environment.

The advantage of EMG signals compared to other means of input is that they form an intuitive interface and they can be used with every patient who is not paralyzed. Even if the muscles are not strong enough or the limbs 
hindered while performing a motion, signals of the intended motion can still be detected.

\section{System Description}

The control system can be divided into four parts: The sensor and actuator hardware, the microcontroller together with the control software, the PC for data visualization and user interaction, and the hardware safety system (refer to Fig. 2).

\section{Sensor and Actuator System}

The sensors integrated for reading the system state and user input are the knee angle sensor (Philips KMZ41 Hall sensor), the six EMG sensors (Delsys 2.3 differential electrodes), and the force sensor (GS Sensors XFTC300) in series with the linear actuator. This actuator consists of a ball screw powered by a standard dc motor (Maxon RE35, $90 \mathrm{~W}$ ) and is driven by a pwm amplifier (Copley 4122Z).

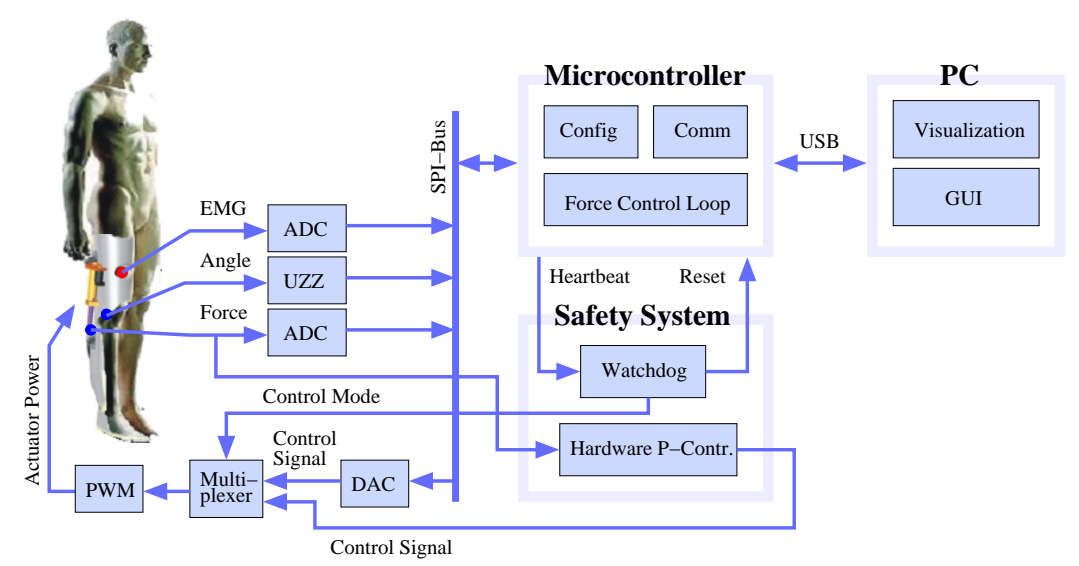

Fig. 2. Hardware of the control system. All sensor data is digitized and transmitted to the microcontroller via SPI bus. In parallel to the microcontroller a safety structure is implemented to control the orthosis in case of a microcontroller failure.

All those sensors and the actuator use converter circuits (MAX1230 ADC, AD5530 DAC, UZZ9001) to communicate with the microcontroller (Atmel Mega 32) via SPI bus. The microcontroller is responsible for taking the sensor information, computing the desired motion and sending appropriate control signals to the pwm amplifier. It also communicates with a PC by USB (nonreal-time) to allow comfortable data visualization and user interaction. 
The EMG signals are taken from the Rectus Femoris, Vastus Medialis, Vastus Lateralis, Semimembranosus, Semitendinosus, and Biceps Femoris. For the actual torque computation only three signals are used (Rectus Femoris, Vastus Medialis, Semimembranosus), the others are recorded to cross-check activation levels during development only.

The EMG signals are post-processed with offset elimination (average value of the last $200 \mathrm{~ms}$ is taken as the offset), rectification and low-pass filtering. A second order Butterworth low-pass filter with a cutoff frequency between $2.0 \mathrm{~Hz}$ to $4.0 \mathrm{~Hz}$ (depending on the experiment and support ratio) was used. The post-processed EMG signal forms the activation envelope of the muscle $j$ and is named $u_{j}(t)$.

\section{Safety System}

The safety system monitors the periodical heartbeat of the microcontroller. In case of a system fault the heartbeat stops and the separate hardware watchdog switches to a secondary controller: This controller is a simple hardware p-controller driven by the force sensor to avoid locking of the knee and allowing free motion (a force value of zero is the target). The EMG sensors have no influence here. The input of the pwm amplifier is switched from microcontroller to hardware p-controller with an analog multiplexer by the watchdog circuit. Switching back to software controlling has to be performed by hand for safety reasons.

\section{Torque Control Loop}

The torque control loop is the central aspect of the control structure. It is responsible for interpreting all necessary sensor data and producing the control value for the pwm amplifier (refer to Fig. 3).

To achieve this, the knee torque resulting from the muscle activations in the human thigh is estimated based on EMG signals. First, the force output for every single muscle $\mathrm{j}$ is calculated by the EMG-to-Force function:

$$
F_{M, j}\left(u_{j}\right)=\frac{e^{A_{j} u_{j} u_{j, \max }^{-1}}-1}{e^{A_{j}}-1} \cdot F_{j, \max },
$$

where $u_{j}$ is the activation of muscle $\mathrm{j}, F_{j \text {, max }}$ is the force output when $u_{j}$ is at its maximum $\left(u_{j}=u_{j, \max }\right)$, and $A_{j}$ is a constant shape factor of the function with $-10 \leq A_{j}<0$. $A_{j}, u_{j, \max }$ and $F_{j, \max }$ are calculated by a calibration routine as described in $[9,10]$.

The resulting knee torque is calculated in the muscle model by

$$
t_{E M G}\left(u_{j}\right)=\sum_{j=1}^{N}\left(\left(\mathbf{I}_{j}-\mathbf{J}\right) \times \frac{\mathbf{I}_{j}-\mathbf{O}_{j}}{\left|\mathbf{I}_{j}-\mathbf{O}_{j}\right|} \cdot F_{M, j}\left(u_{j}\right)\right),
$$

where $\mathbf{J}$ is the vector to the knee joint, $\mathbf{O}_{j}$ and $\mathbf{I}_{j}$ the vectors to origin and insertion of the muscle in the reference frame. Obviously this sum allows cocontraction and co-activation but does not handle them explicitly. 


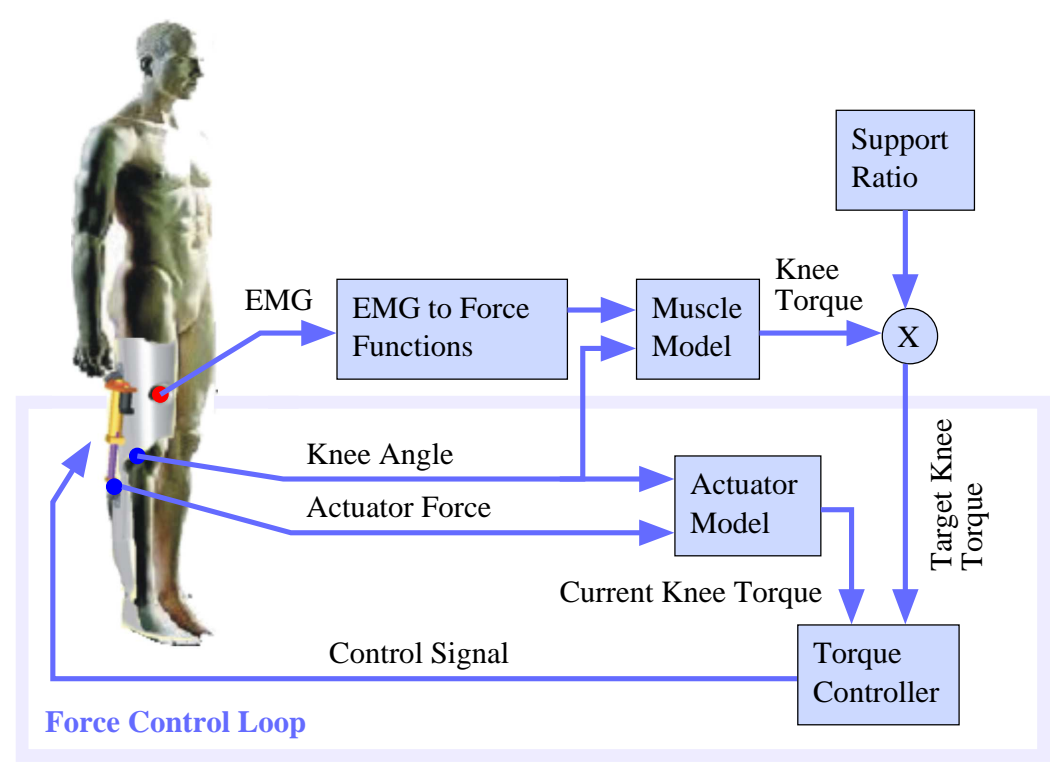

Fig. 3. Force control loop of the microcontroller.

This torque is multiplied by the support ratio $r$ and interpreted as the target torque for the controller: $t_{\text {target }}(t)=t_{E M G}(t) \cdot r$. The current knee torque $t_{\text {cur }}$ is derived from the force sensor that is connected between the actuator and the orthosis by a similar calculation as in Eq. 2. Both torques yield the formula for the control deviation of the torque controller (a standard p-controller to calculate the control signal for the pwm amplifier that drives the actuator): $E=t_{\text {target }}-t_{\text {cur }}$.

\section{Properties of the Torque Control Loop}

This simple control structure has some very interesting properties that will be described here:

For the whole system, only a few sensors are needed. Since human anatomy is very different from subject to subject, the less sensors are necessary the easier it is to adapt to different subjects and perform the calibration.

In contrast to algorithms where a physical model with dynamic equations is implemented, no knowledge about masses or velocities of the body parts is needed. All external and internal forces of the subject are summed up in the force sensor. No explicit measurement or calculation of those quantities is needed to perform the motion calculation. That results in a very robust system with no need for extra sensors for external reaction forces from e.g. the floor, a chair or a hand-rail (which are often difficulty to measure).

Another major difficulty is the unreliability of the EMG signals and the fact that deeper muscles cannot be recorded by surface electrodes. In case of a 
system with a body model that uses position controlled actuators with EMG sensors as the only input, the activation of muscles that are not recorded will not lead to any motion at all: the system will even prohibit the motion. The system described here will also not give any support when the muscles under observation are not activated, but it will not hinder the motion on the other hand, because the torque controller target value will be zero and the orthosis will be moved "out of the way" of the motion. That results in a very sensible behaviour: Given the fact that the controller can move the orthosis as fast as necessary, the exoskeleton will never hinder the subject during arbitrary motions (also if they are induced from outside, like pushing the leg with the hands) and it will support him or her with a specified ratio when muscles under observation are activated. Following that, depending on the activation of the different muscles the orthosis will only change the level of support but it will never hinder any motion.

A drawback of this kind of control loop is, that is is not possible to integrate algorithms for maintaining postural stability of the human. Due to the absence of a dynamic body model, no information about masses, accelerations, and angles is available, making it impossible to set up a system of dynamic equations that could take into account special constraints for the center of gravity and the zero moment point. It is also not possible to predict the motion (or even the direction of the motion) ahead of time to regard constraints like range of the knee angle etc.

\section{Experiments}

The experiments presented here have been performed with a healthy subject that is able to perform all motions on his own accord without any support. Due to safety issues it is currently not possible to perform experiments with patients with disabilities.

Nevertheless the results underline the usefulness of the system. The example motion presented here is climbing a stair. During the first experiment the support ratio was set to zero. The actuator was moving in accordance with the subject's leg, basically neither supporting nor hindering it. Analyzing the result of this setting is very useful to evaluate the controller parameters and the properties of the mechanical construction. Fig. 4 shows the result from this experiment. The knee angle at the beginning is -33deg (0deg means straight leg, negative values indicate knee flexion). After that, the leg is raised to be put on the stair. At $t=1.2 \mathrm{~s}$ the leg is lowered onto the stair and the knee extensor muscles are getting activated. At $t=1.7 \mathrm{~s}$ the knee extensors are strongly activated to step up. At $t=2.4 \mathrm{~s}$ the knee is almost straight again and the activation of the extensor muscles ceases. The torque support of the actuator is almost zero during the whole motion. Except during the quick knee flexion in the interval of $0.7 s<t<1.2 s$ where the actuator is a little too slow to keep up with the intended motion of the subject. A hindering torque 
with a maximum of $5 \mathrm{Nm}$ (positive, indicating knee flexion) is the result. This happens due to the limitation of the actuator current (for safety reasons) and the unfavourable geometric configuration regarding velocity at this point: The higher the knee flexion, the lower the ratio between linear velocity of the actuator and angular velocity of the knee joint (on the other hand the force transmission is higher in this configuration which is very desirable).

Fig. 5 shows the same motion with a support ratio of 2.0. As can be seen, the curve of the knee angle is similar to the one without support, indicating that the system is still controllable by the user and the motion is still as desired. This is a very important fact, since the subject is also able to perform the task on his own. When adding a significant torque to the knee the human brain is fast enough to adapt to the different circumstances and is able to control the intended motion correctly. During push up between $2.0 s<t<2.6 \mathrm{~s}$ the torque produced by the subject's muscles is reduced significantly and the torque contributed by the actuator is roughly two times larger than the estimated value from the human muscles as expected with a support ratio of 2.0 .

Unfortunately it is not easy to compare the sum of the actuator torque and the muscle torque from the first trial with the second trial since different body poses and accelerations in the other joints affect the knee torque.

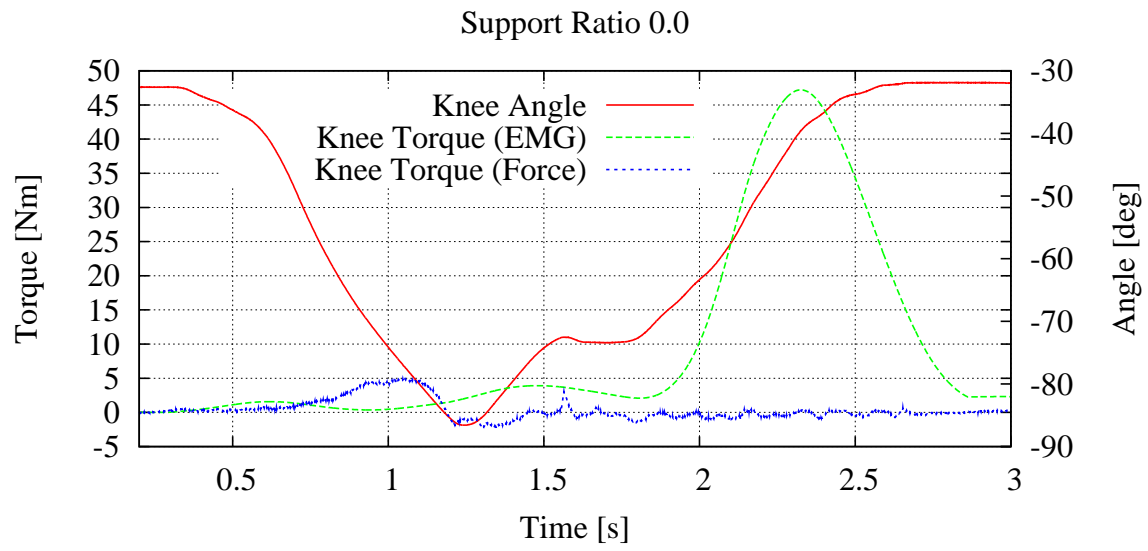

Fig. 4. Stair climbing experiment without support. The knee torque contribution from the actuator as measured by the force sensors is almost zero during the motion.

\section{Results}

The experiments have shown very good results for the stair climbing motion. Another positive example is getting up from a chair or sitting down. Those 


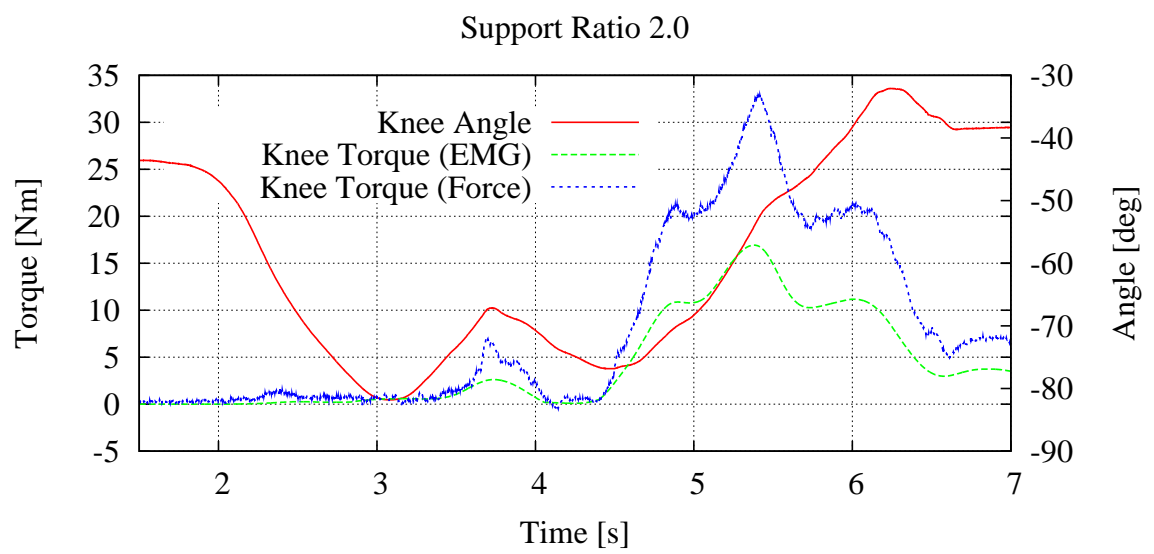

Fig. 5. Stair climbing experiment with $200 \%$ torque support, based on the estimated knee torque derived from the EMG signals.

motions (if executed normally) are not very dynamic and involve strong activation (and easy detection) of relevant muscle groups.

During walking the results are mixed: First of all, during normal walking thigh muscles are activated not very strong. The swinging of the shank is a result of the thigh motion created by the muscles responsible for hip flexion, extension and rotation. Thigh muscles are only used to "guide" the motion, to soften the reversal of motion and to stabilize the knee joint during floor contact. Those activations are very different from the ones during stair climbing and getting up and are harder to support. During floor contact the torque support works fine, but during the swing phase it rather leads to a motion that is not as smooth as desired. It might be sensible to turn off the force support during swing-phase. More work has to be performed in this area.

\section{Conclusion}

In this paper a system was presented to control an exoskeleton leg. Similar to the work in [8], the control loop utilizes a torque controller to add an adjustable support for the subject wearing the exoskeleton.

As shown with the experiments, using EMG signals as an input interface for a lower leg exoskeleton produces promising results for further investigations. They offer an intuitive way of commanding a control structure with the restriction that the user must be able to activate the muscles in a proper way to perform the desired motion.

The next steps of research will include making the motions more smooth and the EMG input safer by adding control layers to cope with undesired 
bursts. Once this and other safety features are implemented, experiments with patients can be performed.

\section{References}

1. H. Kazerooni, J.-L. Racinea, L. Huang, and R. Steger, "On the control of the berkley lower extremity exoskeleton (bleex)," in Proceedings of the 2005 IEEE International Conference on Robotics and Automation, 2005, pp. 4353-4360.

2. H. Kawamoto, S. Lee, S. Kanbe, and Y. Sankai, "Power assist method for hal-3 using emg-based feedback controller," in Proceedings of the IEEE International Conference on Systems, Man and Cybernetics, vol. 2, 2003, pp. 1648-1653.

3. G. S. Sawicki, K. E. Gordon, and D. P. Ferris, "Powered lower limb orthoses: Applications in motor adaption and rehabilitation," in Proceedings of the IEEE International Conference on Rehabilitation Robotics, vol. 2, 2005, pp. 206-211.

4. S. Lee and G. N. Sridis, "The control of a prosthetic arm by EMG pattern recognition," in IEEE Transactions on Automatic Control, vol. AC-29, no. 4, 1984, pp. 290-302.

5. O. Fukuda, T. Tsuji, H. Shigeyoshi, and M. Kaneko, "An EMG controlled human supporting robot using neural network," in Proceedings of the IEEE/RSJ Int. Conf. on Intelligent Robots and Systems, 1999, pp. 1586-1591.

6. S. Morita, T. Kondo, and K. Ito, "Estimation of forearm movement from EMG signal and application to prosthetic hand control," in Proceedings of the IEEE Int. Conf. on Robotics ES Automation, 2001, pp. 3692-3697.

7. D. G. Lloyd and T. F. Besier, "An emg-driven musculoskeletal model to estimate muscle forces and knee joint moments in vivo," Journal of Biomechanics, vol. 36, pp. $765-776,2003$.

8. J. Rosen, M. Brand, M. B. Fuchs, and M. Arcan, "A myosignal-based powered exoskeleton system," in IEEE Transactions on Systems, Man, and Cybernetics, vol. 31, 2001.

9. C. Fleischer, K. Kondak, C. Reinicke, and G. Hommel, "Online calibration of the emg-to-force relationship," in Proceedings of the IEEE/RSJ Int. Conf. on Intelligent Robots and Systems, 2004.

10. C. Fleischer and G. Hommel, "Predicting the intended motion with emg signals for an exoskeleton orthosis controller," in Proceedings of the IEEE/RSJ Int. Conf. on Intelligent Robots and Systems, 2005. 